This item was submitted to Loughborough's Research Repository by the author.

Items in Figshare are protected by copyright, with all rights reserved, unless otherwise indicated.

\title{
Mesoscopic structure features in synthetic graphite
}

PLEASE CITE THE PUBLISHED VERSION

https://doi.org/10.1016/j.matdes.2018.01.038

PUBLISHER

(c) Elsevier

VERSION

AM (Accepted Manuscript)

\section{PUBLISHER STATEMENT}

This work is made available according to the conditions of the Creative Commons Attribution-NonCommercialNoDerivatives 4.0 International (CC BY-NC-ND 4.0) licence. Full details of this licence are available at: https://creativecommons.org/licenses/by-nc-nd/4.0/

\section{LICENCE}

CC BY-NC-ND 4.0

\section{REPOSITORY RECORD}

Maerz, Benjamin, Kenny Jolley, Thomas James Marrow, Zhaoxia Zhou, Malcolm Heggie, Roger Smith, and Houzheng Wu. 2018. "Mesoscopic Structure Features in Synthetic Graphite”. Loughborough University. https://hdl.handle.net/2134/31991. 


\title{
Mesoscopic structure features in synthetic graphite
}

\author{
Benjamin März a , Kenny Jolley ${ }^{\mathrm{b}}$, Thomas James Marrow ${ }^{\mathrm{c}}$, Zhaoxia Zhou ${ }^{\mathrm{a}}$, \\ Malcolm Heggie ${ }^{\mathrm{b}}$, Roger Smith ${ }^{\mathrm{d} *}$, Houzheng $\mathrm{Wu}^{\mathrm{a}^{*}}$ \\ ${ }^{a}$ Department of Materials, Loughborough University, Leicestershire, LE11 3TU, UK \\ ${ }^{\mathrm{b}}$ Department of Chemistry, Loughborough University, Leicestershire, LE11 3TU, UK \\ ${ }^{\mathrm{c}}$ Department of Materials, University of Oxford, Parks Road, Oxford, OX1 3PH, UK \\ ${ }^{\mathrm{d}}$ Department of Mathematical Science, Loughborough University, Leicestershire, LE11 3TU, \\ $U K$
}

\begin{abstract}
The mesocopic structure features in the coke fillers and binding carbon regions of a synthetic graphite grade have been examined by high resolution transmission electron microscopy (TEM) and Raman spectroscopy. Within the fillers, the three-dimensional structure is composed of crystal laminae with the basal plane dimensions $\left(\mathrm{L}_{\mathrm{a}}\right)$ of hundreds nanometres, and thicknesses $\left(\mathrm{L}_{\mathrm{c}}\right)$ of tens of nanometres. These laminae have a nearly perfect graphite structure with almost parallel c-axes, but their a-b planes are orientated randomly to form a “crazy paving” structure. A similar structure exists in the binding carbon regions, with a smaller $\mathrm{L}_{\mathrm{a}}$. Significantly bent laminae are widely seen in quinoline insoluble inclusions and the graphite regions developed around them. The $\mathrm{L}_{\mathrm{a}}$ values measured by TEM are consistent with estimates from the intensity ratios of the $D$ to $G$ Raman peak in these regions. Atomistic modelling finds that the lowest energy interfaces in the crazy paving structure comprise 5, 6 and 7 member carbon rings. The bent laminae tend to maintain the 6 member rings, but are strained elastically. We suggest that a 7 member carbon ring leaves a cavity representing an arm-chair graphite edge contributing to the Raman spectra D peak.
\end{abstract}

Key Words: graphite; graphene; mesoscopic structure; crazy paving; carbon ring; elastic strain

\footnotetext{
*Corresponding author.

Telephone: +44 1509223342

E-mail address: h.wu2@lboro.ac.uk (Houzheng Wu), roger.smith@lboro.ac.uk (Roger Smith)
} 


\section{Introduction}

Graphite manufactured by isostatic pressure moulding, often called isotropic graphite or isographite, is the likely new generation nuclear graphite for GEN-III/GEN-IV high temperature reactors (HTR) [1], [2]. This type of graphite is also widely used in the manufacturing of semiconductor and photovoltaic materials where high purity and excellent mechanical and thermal properties are required. Compared to currently applied coarse grained graphites manufactured by extrusion or vibration moulding, iso-graphite generally comprises of much finer coke particles in the tens of microns range, instead of hundreds or even thousands of microns [3]. To produce the micro-scale coke filler of the required size and size distribution, substantial milling of the coke is necessary, including intensive mixing with the binding pitch to plasticise the filler powder. This enables it to be moulded into the required shapes and sizes that confer sufficient strength in the final product [4]. Therefore, one may expect that the structure developed in coke filler and pitch binder may be different from that observed in traditionally prepared graphites [5], [6]. More importantly, one needs to know how the structural features at all length scales influence the physical properties, and engineering performance, such as neutron irradiation damage and oxidation resistance. Success in establishing such relationships can more scientifically guide the design of formulation and selection of processing conditions in graphite manufacture.

Several research groups have examined the structure of traditional and new graphite grades engineered for nuclear applications [5], [7]-[9], including iso-graphite, mainly using transmission electron microscopy (TEM). In particular, Karthik et al. [9] compared the structures of next generation graphites and concluded that all were composed of a turbostratic structure in which "close-packed carbon planes are curled and rotated with respect to each other". They proposed that "the atoms are arranged in layers similar to graphite, but stacked randomly”. Wen et al. [10] reported further structural features as nano-sized graphite structures, chaotic structures and non-graphitising carbon in the binder of Gilsocarbon and PGA (Pile Grade A) graphites. Inside the binder, the most notable features are the quinoline insoluble (QI) particles, which have been widely observed in all graphites. QI particles are supposed to originate from carbon blacks, coke, coal dusts and cenospheres [11] which are considered as inert material, and may have a beneficial effect in reducing the effect of temperature on the viscosity variation of pitch [12]. The structure inside the QI is composed of nano-sized crystallites of graphite. Together with existing microcracks and pores inside graphite, these crystalline features are believed to exert a strong influence, not only on the 
mechanical and physical properties of a graphite grade, but also on its neutron irradiation damage behaviour [13].

It is well known that neutron damage varies between graphite grades [14], and this may be due to differing atomic structure. The sensitivity to atomic structure has been illustrated by recent molecular dynamic studies [15] that found channelling of knock-on atoms. To elucidate the neutron irradiation damage mechanism of an industrial nuclear graphite grade, it is therefore necessary to establish first the "turbostratic structure" and the nanostructure inside a nuclear graphite before irradiation. In this study, we determine the micro- and mesostructures in an iso-graphite grade using TEM, high resolution TEM (HRTEM) and Raman spectroscopy. The key structural features observed experimentally are then modelled in atomistic detail using atomistic modelling.

\section{Experimental procedures}

\subsection{Material and sample preparation}

The graphite material (grade SNG623) supplied by Sinosteel Advanced Materials Ltd is one of the experimental grades developed for a neutron irradiation programme that is being conducted at the Oak Ridge National Laboratory, USA [16], [17]. A highlight on the manufacture of this grade and its representative properties are available in the Data in Brief, (DiB) [18] .

All samples used in this study were machined from the same billet as the test samples for the accompanying neutron irradiation programme. The as-received samples are all in the form of circular tablets with a diameter of $10 \mathrm{~mm}$ and thickness $0.5 \mathrm{~mm}$. The as-machined surface is known to be deformed and is not representative of the bulk [19], so a fracture surface of an as-received sample was used. The examined fracture surfaces were prepared in a controlled manner by breaking the as-received tablets manually. A graphite tablet was positioned over a small breaker bar to define the fracture location and a flat steel ruler was pressed on top of it with a bending force applied, until the sample broke. These as-fractured surfaces were examined with scanning electron microscopy (SEM) and Raman spectroscopy to select regions of interest for analysis. Samples for TEM and HRTEM examination were extracted from the fracture surface using the procedures described below. 
TEM specimens were prepared using a focussed ion beam (FIB) in a FEI Nova 600 Nanolab Dual Beam system. This method allowed the preparation of a TEM specimen from a position of particular interest on the fracture surface, e.g. from a selected coke filler or binding carbon. Details of the TEM sample preparation are available in the DiB [18].

\subsection{Structure characterisation}

\section{(1) Raman Spectroscopy}

Raman spectroscopy was conducted using a Horiba Jobin-Yvon LabRam HR high spectral resolution Raman system with an integral confocal microscope, at a laser wavelength of $514 \mathrm{~nm}$ (green). A highlight of the measuring procedure and spectrum analysis is available in the $\mathrm{DiB}[18]$.

\section{(2) SEM and TEM examination}

The morphology of the fracture surface is imaged using secondary electrons in a field emission gun SEM (FEG-SEM) (JEOL 7800F, Tokyo, Japan) at an acceleration voltage of $1 \mathrm{kV}$. TEM/HRTEM was conducted with a FEI Tecnai F20 microscope operated at $80 \mathrm{kV}$. High resolution annular dark-field (ADF) scanning transmission electron microscopy (STEM) was carried out using an aberration corrected $\left(\mathrm{C}_{s}\right)$ JEOL JEM-ARM200F microscope at $80 \mathrm{kV}$. In ADF STEM a focussed electron beam is scanned over the specimen in a raster, while the transmitted scattered intensity is collected on an annular detector, which is arranged around the optical axis. ADF STEM allows easier image interpretation due to no contrast reversals and delocalisation compared to HRTEM [20, p. 104].

\section{Experimental Results}

\subsection{Morphology of as-fractured graphite}

An overview of an as-fractured surface is shown in Figure 1(a). There are characteristic regions that can be described as: flat and smooth, labelled "F"; rounded and rough, labelled "B"; and voids, labelled "V". Most areas on the fracture surface show the morphology labelled "B" and only small regions are characteristic of those labelled " $F$ ". The flat " $F$ " regions are exposed pre-existing cracks within filler particles [21], and are unaffected by the fracturing of the specimen. Voids exist everywhere, but it is difficult to differentiate those formed during manufacture from those generated by dislodging of fillers during fracture. More details of key features on the as-fractured surface are highlighted in the DiB [18]. F-1 and F-2 regions are defined as filler surface without binding carbon, and a filler subjected to 
trans-granular fracture, respectively. B-1 and B-2 regions are defined as exposed filler with patched binding carbon attached, and binding carbon only, respectively.

These observations show that the surface of as-fractured graphite exposes the binding matrix and course fillers and allows them to be identified. The following analysis will focus on the mesoscopic structure inside the filler and binding carbon, obtained by sampling different regions on a fracture surface.

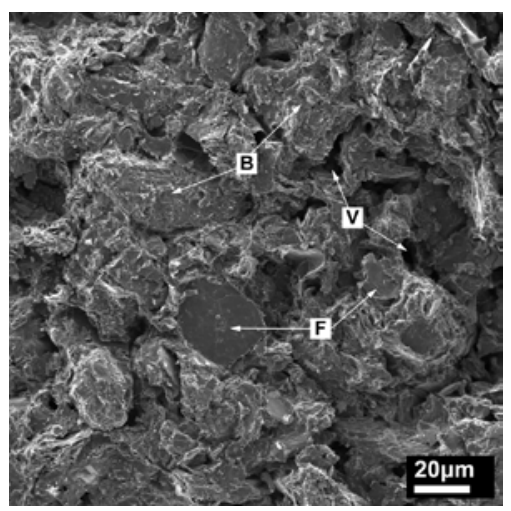

Figure 1 Overview of the morphologies of the asfractured surface of isostatically moulded graphite by snapping a sample. F, B and V represent filler, binding carbon and void, respectively. More details of each region are available in the $\mathrm{DiB}[18]$.

\subsection{Disordered structure measured by Raman spectroscopy}

Representative Raman spectra of SNG623 acquired through randomly probing an asfractured surface and of HOPG are shown in the DiB [18], including description of the key features.
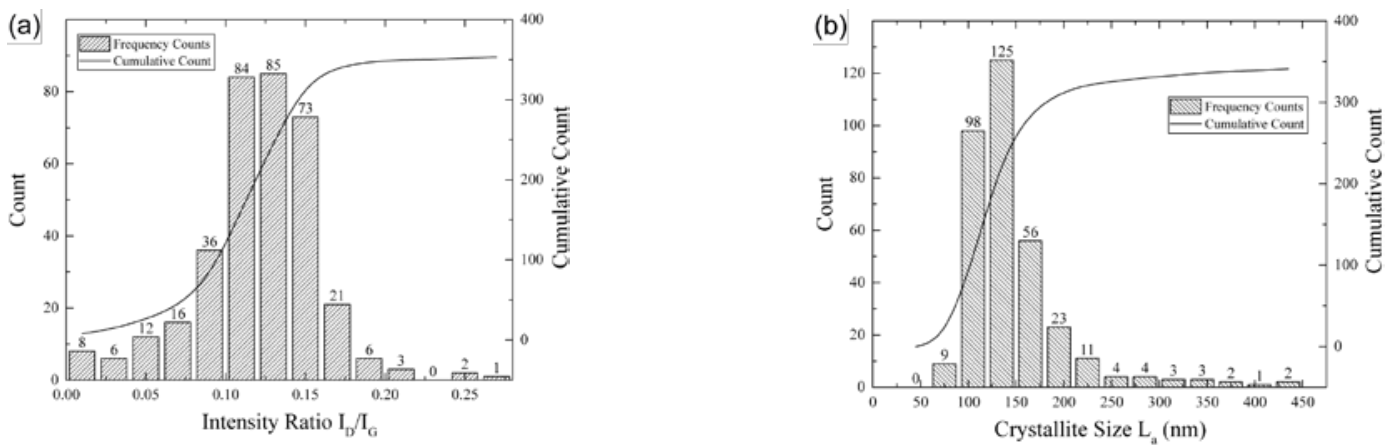

Figure 2 Raman measurement on an as-fractured surface of SNG623: histogram distribution of (a) $\mathrm{I}_{\mathrm{D}} / \mathrm{I}_{\mathrm{G}}$ ratios and (b) $\mathrm{L}_{\mathrm{a}}$ of graphite crystallites determined using Equation 1.

The intensity ratio of the $\mathrm{D}$ and $\mathrm{G}$ bands $\left(\mathrm{I}_{\mathrm{D}} / \mathrm{I}_{\mathrm{G}}\right)$ has been empirically demonstrated to be inversely proportional to the in-plane graphite crystallite sizes $L_{a}(\mathrm{~nm})$, [22]-[24] i.e.

$$
L_{a}=\left(2.4 \times 10^{-10}\right) \lambda^{4}\left(\frac{I_{D}}{I_{G}}\right)^{-1}
$$


where $\lambda$ is the wavelength of the excitation laser $(\mathrm{nm})$. A histogram distribution of the measured $I_{D} / I_{G}$ intensity ratios on as-fractured surfaces are summarised in Figure 2(a), and the corresponding histogram of $\mathrm{L}_{a}$ is shown in Figure 2(b). The measurements show that among the probed positions the true range of $\mathrm{L}_{a}$ is $62-440 \mathrm{~nm}$, with an average $\mathrm{L}_{a}$ of $147.7 \pm 54.4 \mathrm{~nm}$.

To link an $I_{D} / I_{G}$ measurement, or estimated $L_{a}$ value, to a specific region on the fracture surface, a fracture surface area was scanned using the Raman probe, followed by imaging with SEM to match the visible features with the $L_{a}$ values. The results are summarised in Figure 3. Figure 3(a) shows the overall view of the variation of $L_{a}$ across the scanned area. It is noted that there are 5 localised regions in this area where the $L_{a}$ value has its highest extrema: $667.6 \mathrm{~nm}, 662.4 \mathrm{~nm}, 537.7 \mathrm{~nm}, 513.9 \mathrm{~nm}$ and $458.1 \mathrm{~nm}$. The diameters of these regions lie between 5 and $15 \mu \mathrm{m}$. By overlaying the SEM image on the $\mathrm{L}_{\mathrm{a}}$ map, it is shown that these regions with high $\mathrm{L}_{\mathrm{a}}$ match very well with the regions having features such as $\mathrm{F}-2$, i.e. a fracture surface of a filler (Figure 1(d) in [18]). The rest of the $L_{a}$ map is dominated by $\mathrm{L}_{\mathrm{a}}$ with an average of $109.9 \pm 38.4 \mathrm{~nm}$ and morphology features similar to B-2 [18] . Intermediate $L_{a}$ values, all within the largest $5 \%(\geq 176.9 \mathrm{~nm})$, are related to some dappled regions, which we believe are likely linked to regions with features B-1 [18]).

As per the matching results shown in Figure 3, we can point out that, for the histogram distribution shown in Figure 2, the estimated $L_{a}$ values on the far left-hand side should correspond to graphite crystallites in binding carbon with the values on the far right-hand side representing those in coke fillers. Intermediate values possibly belong to a mixture of binding carbon and fillers, since the Raman laser probes a small but distinct area on the sample, and may overlap filler and binding carbon. The peak of the histogram distribution appears near the lower bound, implying graphite crystallites in binding carbon may be the main contributor. This is to be expected if the majority of the fracture surface exposes binding carbon.

Pimenta et al. [22] experimentally demonstrated that the intensity of the $\mathrm{D}$ band shows a strong correlation with the edge structure of a graphite crystallite. Between the two types of edge structures (armchair and zigzag), they suggested that the former has a much stronger influence than the latter on the intensity of the $\mathrm{D}$ band. If so, the graphene sheets in the graphite crystallites must generally be joined with the armchair structure, as otherwise it would be impossible to see the difference in $\mathrm{L}_{\mathrm{a}}$. Pending further experimental evidence, this 

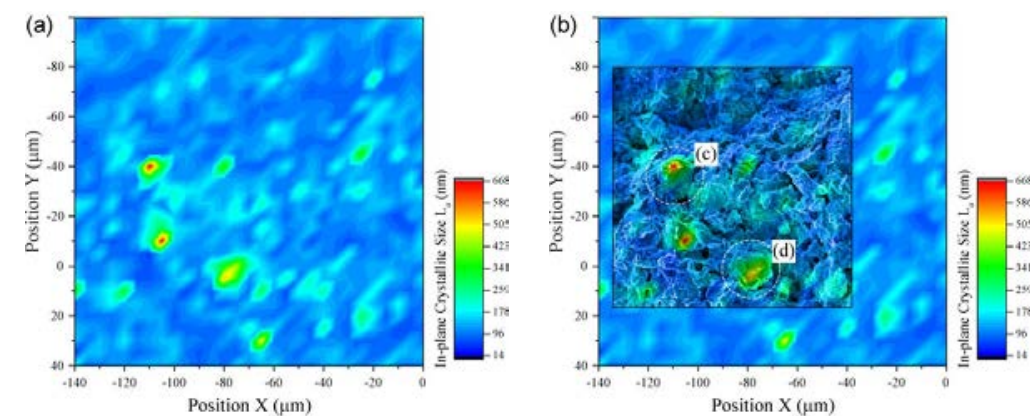

Figure $3 \quad$ (a) Map of $\mathrm{La}_{\mathrm{a}}$ estimated from the $\mathrm{I}_{\mathrm{D}} / \mathrm{I}_{\mathrm{G}}$ ratio on an as-fractured surface. (b) Correlation of the SEM image of the fracture surface, overlaid on the $\mathrm{L}_{\mathrm{a}}$ map, showing a typical cleavage plane. (c) \& (d) SEM images of the matching locations
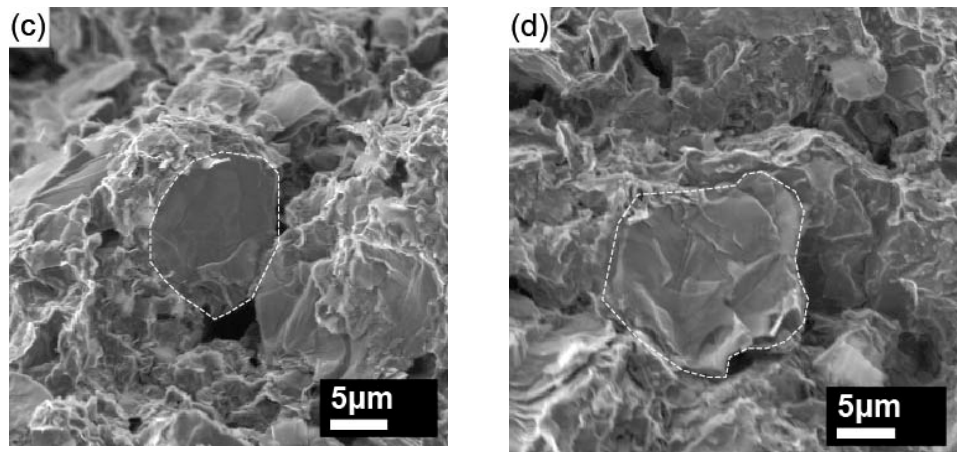
indicated in (b), giving $\mathrm{L}_{\mathrm{a}}$ values of $662.4 \mathrm{~nm}$ and $537.7 \mathrm{~nm}$.

hypothesis is tested by atomistic modelling, supported by results of the structural analysis by means of TEM, as presented in section 4.

From the Raman mapping results, we can claim that the coke fillers have graphite crystallites with $L_{a}$ values larger than those in binding carbon, by a factor of a few times up to an order of magnitude.

\subsection{Structure of graphite analysed by TEM}

\section{(1) Structure underneath an F region}

TEM observations from an $\mathrm{F}$ region are presented in Figure 4. Figure 4(a) shows the sampling position on a region with $\mathrm{F}$ type surface morphology, and Figure 4(b) shows the SEM image of a cross-sectional TEM foil lifted out from this position. In Figure 4(c) a TEM bright field (BF) image showing an overview of a cross-section of the cleavage plane of a coke filler is presented, taken at $0^{\circ}$ sample tilt and rotation. There is a clear diffraction contrast, evidencing that this region is not a single crystal. In fact, this diffraction contrast implies that a highly-textured lamina structure exists inside this filler. Following the bent contour of the fracture surface, the laminae are bent in the same manner.
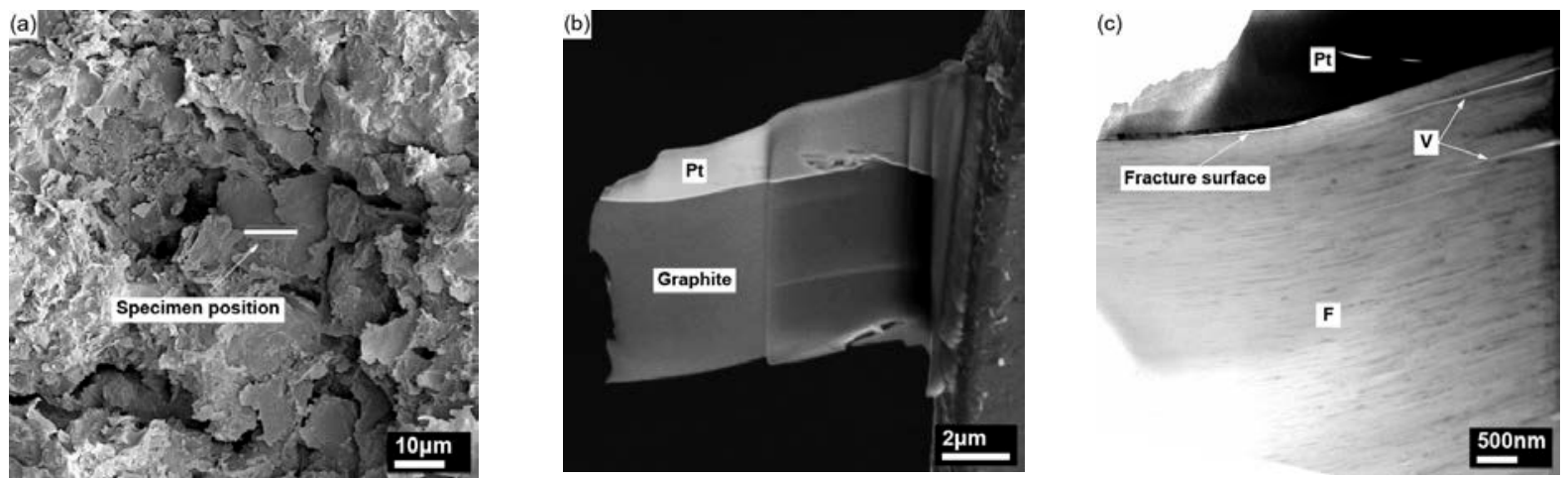

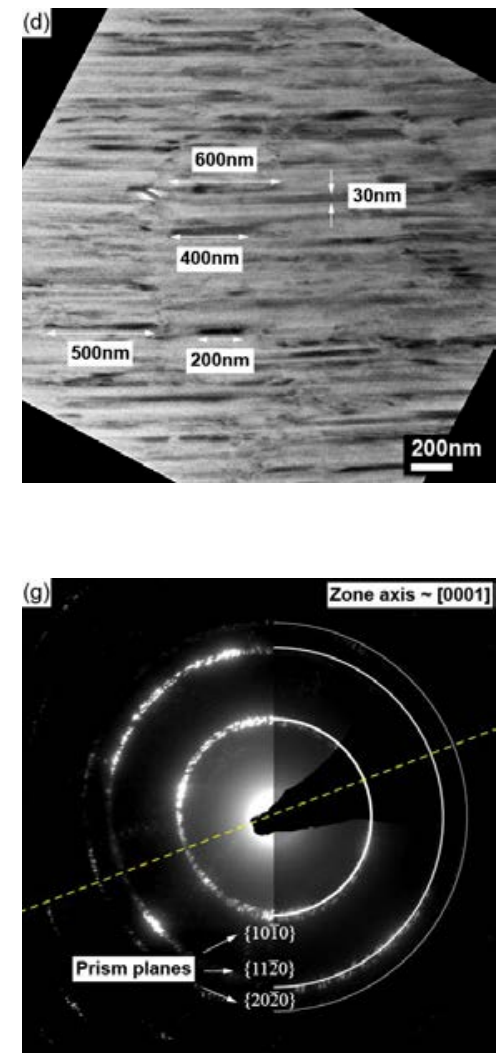
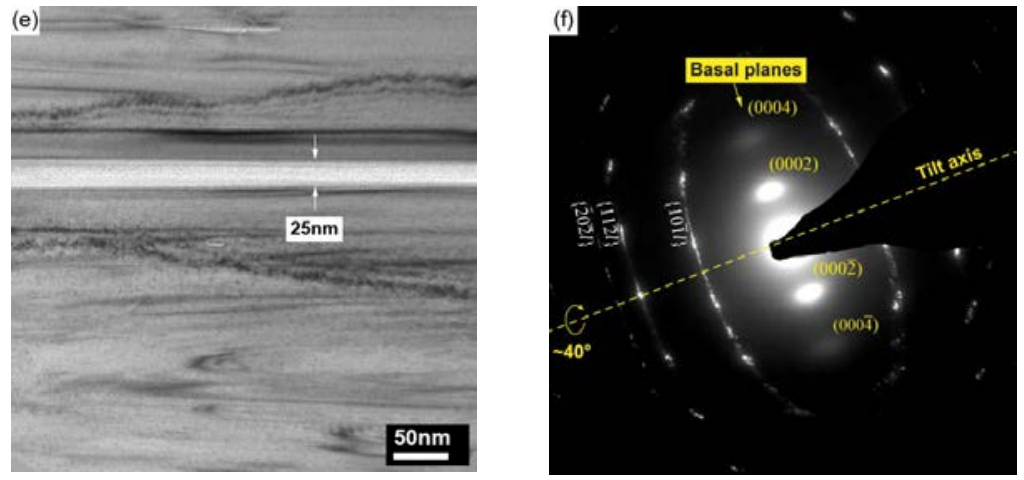

Figure 4 TEM examination of a filler with specimen foils prepared by FIB. (a) Fracture surface with the TEM sampling position labelled; (b) SEM image of the asprepared TEM foil; (c) TEM BF images showing a lamina structure; (d) TEM BF view of the lamina structure showing laminae of typical lengths of 200 to $600 \mathrm{~nm}$ and thickness around $30 \mathrm{~nm}$ in this filler; (e) a crack proceeding parallel to the laminae; (f) SAED pattern of the untilted specimen; and (g) SAED pattern at $\sim 40^{\circ}$ sample tilt.

The contrast between adjacent laminae indicates that they have different orientations. Along a lamina, the contrast alters without an obvious periodicity, implying that each lamina is composed of multiple segments with different in-plane crystal orientation. To measure the dimensions of the resolved laminae, a higher magnification BF image is presented in Figure 4(d). The thickness of the diffracting domains (or laminae), as measured by random sampling of 30 positions, is $21.1-44.6 \mathrm{~nm}$ with an average of $30.9 \pm 6.6 \mathrm{~nm}$. The lengths along the longitudinal direction of these diffracting domains have a broad distribution from a few nanometres up to about a thousand nanometres, but are quite hard to measure due to overlapping boundaries. Some of the measurements are indicated exemplarily in Figure 4(d). This length distribution may partly be a consequence of cross-sectioning at different positions of each lamina. The domains within a lamina likely have near polygonal shapes, as well as size differences. The in-plane average dimension of these laminae ought to be several hundred nanometres with a similar range of $\mathrm{L}_{\mathrm{a}}$ as estimated using the $\mathrm{I}_{\mathrm{D}} / \mathrm{I}_{\mathrm{G}}$ ratio determined by Raman spectroscopy in the F regions.

Micro-cracks are present inside this region as shown in Figure 4(c). An image with higher magnification of a micro-crack is shown in Figure 4(e). This type is believed to be a Mrozowski crack, as widely reported [6], [7], [9], [25], [26], [27]-[29]. Such cracks are formed during manufacturing when a billet is cooled down after graphitisation or after coking, 
a step involved in raw material preparation. It is noted that a layer of amorphous carbon remains on the crack surface. Whilst there is a possibility that it may be formed by the redeposition during TEM sample preparation using FIB, others have observed a similar material filling a micro-crack in a TEM sample prepared from traditional Ar ion thinning techniques [6], [9].

Selected area electron diffraction (SAED) patterns acquired from a region in Figure 4(c) are given in Figure 4(f, g). The SAED pattern shown in Figure 4(f) was taken without tilting the specimen. Elongated spots or arcs arise, lying on different ellipses. This is an indication of a textured polycrystalline structure with a texture axis inclined to the electron beam [30, p. 273]. Such deviation from a shared texture axis is a consequence of TEM sample preparation when the FIB cross-sectioning was made along an oblique direction with respect to the lamellar crystal structure. As graphite has a plate texture, the texture axis points along the [0001] direction. In the SAED, the elongated spots on each single ellipse result from different Laue zones and can be indexed using the same hki indices but a different $l$ [30, p. 274]. Consequently, the indices of the ellipses were determined to be $\{10 \overline{1} l\},\{11 \overline{2} l\}$ and $\{20 \overline{2} l\}$, while $\{10 \overline{1} 0\},\{11 \overline{2} 0\}$ and $\{20 \overline{2} 0\}$ belong to the prism planes. On an imaginary line passing through the central spot, much brighter diffraction spots arise in the pattern in Figure 4(f). They belong to the graphite basal planes and since they result from different zones as well, they have to be indexed as (000l). By tilting the specimen by $\sim 40^{\circ}$ the texture axis and thus the [0001] crystallite directions have been oriented almost parallel to the electron beam. Consequently, only diffraction rings resulting from the prism planes (hki0) arise in this SAED pattern, as shown in Figure 4(g). The missing (000l) diffraction rings are the only indication of a textured polycrystalline structure. The diffraction rings are nearly continuous since the diffracting domains within the laminae are randomly rotated around the texture axis, i.e. the [0001] crystal direction. Broadening of the diffraction rings in turn is a combined result. In the first place there are slight variations of the normal direction of the diffracting domains caused by the bent laminae. Thus, by tilting the specimen in the microscope, the texture axis could not be aligned perfectly parallel to the electron beam for all the domains. In addition, the small crystallite/domain size may make a minor contribution due to Scherrer broadening.

To examine the in-plane shapes of the laminae in a coke, a TEM specimen was prepared from a coke grain in the way that the [0001] crystallite directions are aligned close to the electron beam direction, as detailed in the $\mathrm{DiB}$ [18]. A representative TEM BF image is shown in 
Figure 5(a). The shadows of some laminae can be differentiated by diffraction contrast as well as moiré patterns that appear as a result of superimposed hexagonal structures. The inplane shape of the resolved laminae is outlined in Figure 5(b); they all have different polygonal shapes. It is noted that some polygons are overlapped; this is likely due to the TEM sample being thicker than most of the laminae and thus the projection of more than one lamina is recorded in the final image in most of the examined area. Figure 5(c) shows the SAED pattern of nearly the whole region shown on Figure 5(a) and (b). Diffraction rings of only the prismatic planes confirm that the graphite crystallites within the laminae are textured along [0001]. The sharpness of the rings indicates almost no deviation from the texture axis.

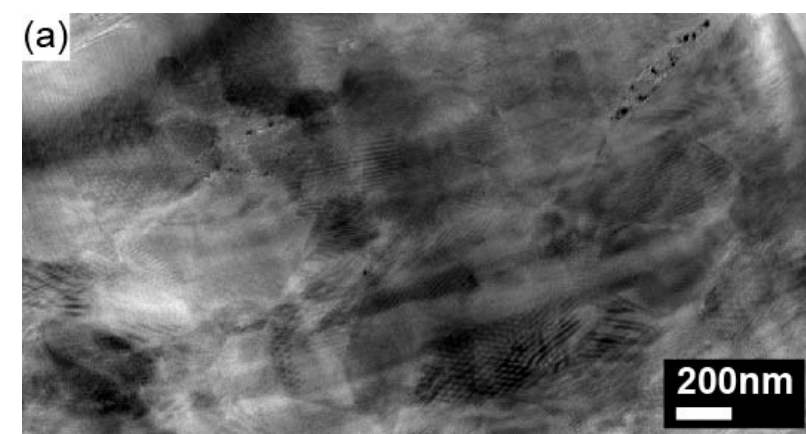

(b)

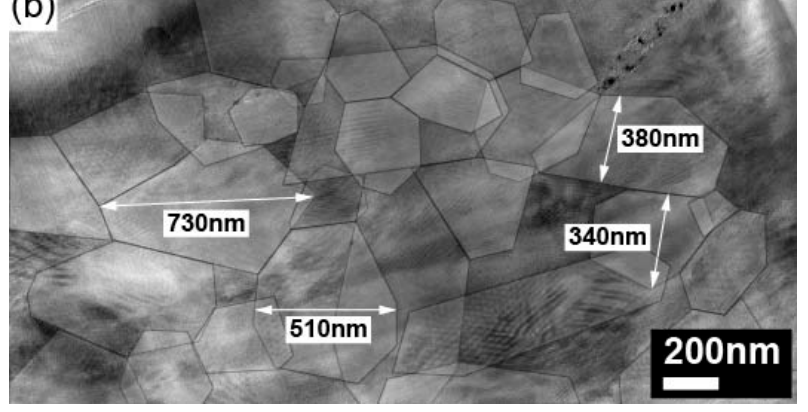

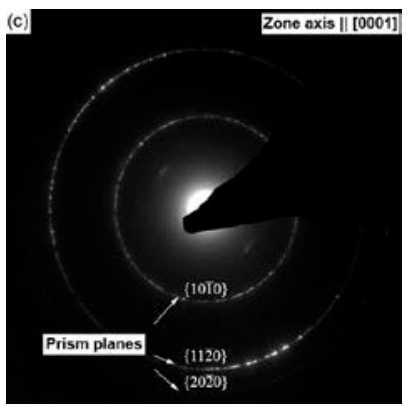

Figure 5 Structure of a coke filler imaged along the normal direction of laminae. (a) TEM BF image; (b) polygonal shape of each lamina outlined. (c) Selected area diffraction pattern of the region shown in (a).

\section{(2) Structure underneath a B region}

Specimens for TEM analysis of binding carbon were prepared from a location showing typical morphology of region B, as exemplified by Figure 6(a). A TEM sample lifted out from the position shown in Figure 6(a) is presented in Figure 6(b). Another TEM sample lifted from a similar position is presented in Figure 6(d). The overview of the microstructure of each of the two TEM samples is shown in Figure 6(c) and (e). In the regions of binding carbon, the following features are clearly identified, as observed by other researchers [5], [6], [9], [28]: graphite crystallites, QI particles, micro-cracks and pores as well as interfaces with coke fillers. Details of these features are presented below.

\section{(a) Graphite crystallites}

Judging from the TEM diffraction contrast images and SAED pattern acquired from the graphite crystallites presented in different binder regions, there is no clear difference in 
graphite structure, compared to that in the coke filler, as shown in section (3.3-1). The graphite crystallites exist in a laminated fashion with a shared axis along the [0001] direction, but the laminae are bent and folded in several directions. Such unique structures can be seen from Figure 6(c) where the graphite regions were developed around the QI particles. A similar structure was observed by other researchers [7]-[9].

Figure 6(g) shows another type of graphite crystallite, labelled as 1 and outlined by a dashed line. It is surrounded by regions dispersed with QI particles, labelled as 2 and 3. It shows similar laminate structure as observed in a coke filler, and measurements of $\mathrm{L}_{\mathrm{a}}$ are around a few hundred nanometres.
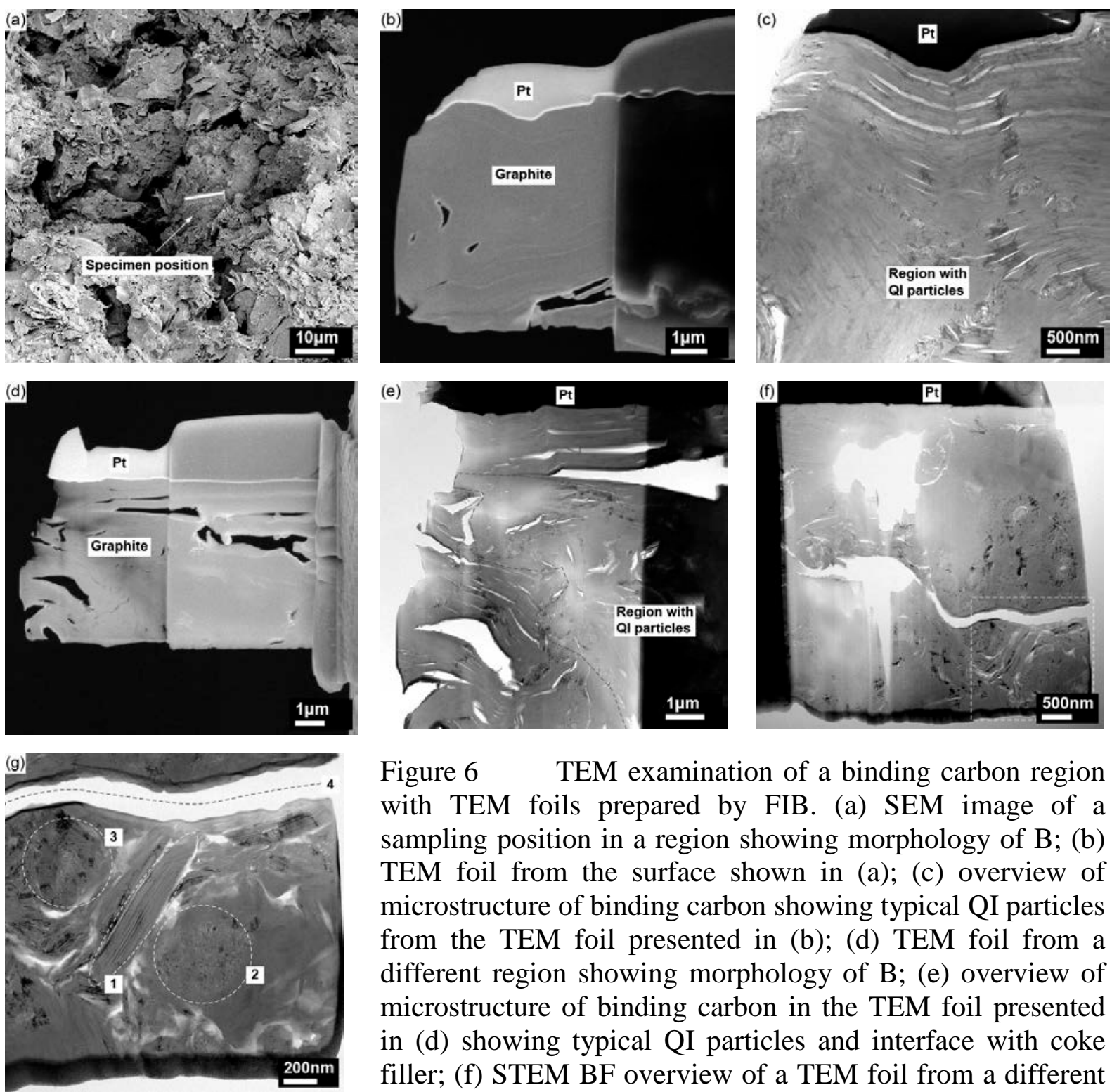

Figure 6 TEM examination of a binding carbon region with TEM foils prepared by FIB. (a) SEM image of a sampling position in a region showing morphology of B; (b) TEM foil from the surface shown in (a); (c) overview of microstructure of binding carbon showing typical QI particles from the TEM foil presented in (b); (d) TEM foil from a different region showing morphology of B; (e) overview of microstructure of binding carbon in the TEM foil presented in (d) showing typical QI particles and interface with coke filler; (f) STEM BF overview of a TEM foil from a different region of binding carbon; (g) STEM BF view of the microstructure showing a well crystallised particle with nanocracks (1) besides QI particles with chaotic (2) and rosette (3) structures as well as a large crack (4). 


\section{(b) Boundaries}

It is difficult to characterise the boundary between coke filler and binding carbon due to the lack of a clearly defined interface. Figure 6(e) shows a region where a boundary between a filler and the binding carbon region is approximately outlined based on the fact that QI particles are commonly present in binding carbon, but not in the filler. There are other boundaries between graphite particles shown in Figure 6(g) around region 1, where the graphite regions with different orientation are coherently bonded together. They are similar to grain boundaries or large angle interfaces in polycrystalline metals or ceramics.

\section{(c) QI particles}

Through a statistical analysis of two specimens, the sizes of QI particles vary from 84.7 $869.3 \mathrm{~nm}$ in diameter with an average value of $259.0 \pm 166.7 \mathrm{~nm}$. TEM images show that they are not evenly distributed in a binding carbon region. The average density of QI particles is about 1.5 particles per $\mu \mathrm{m}^{2}$, estimated by counting 80 QI particles within an area of $53.5 \mu \mathrm{m}^{2}$ of binding carbon.
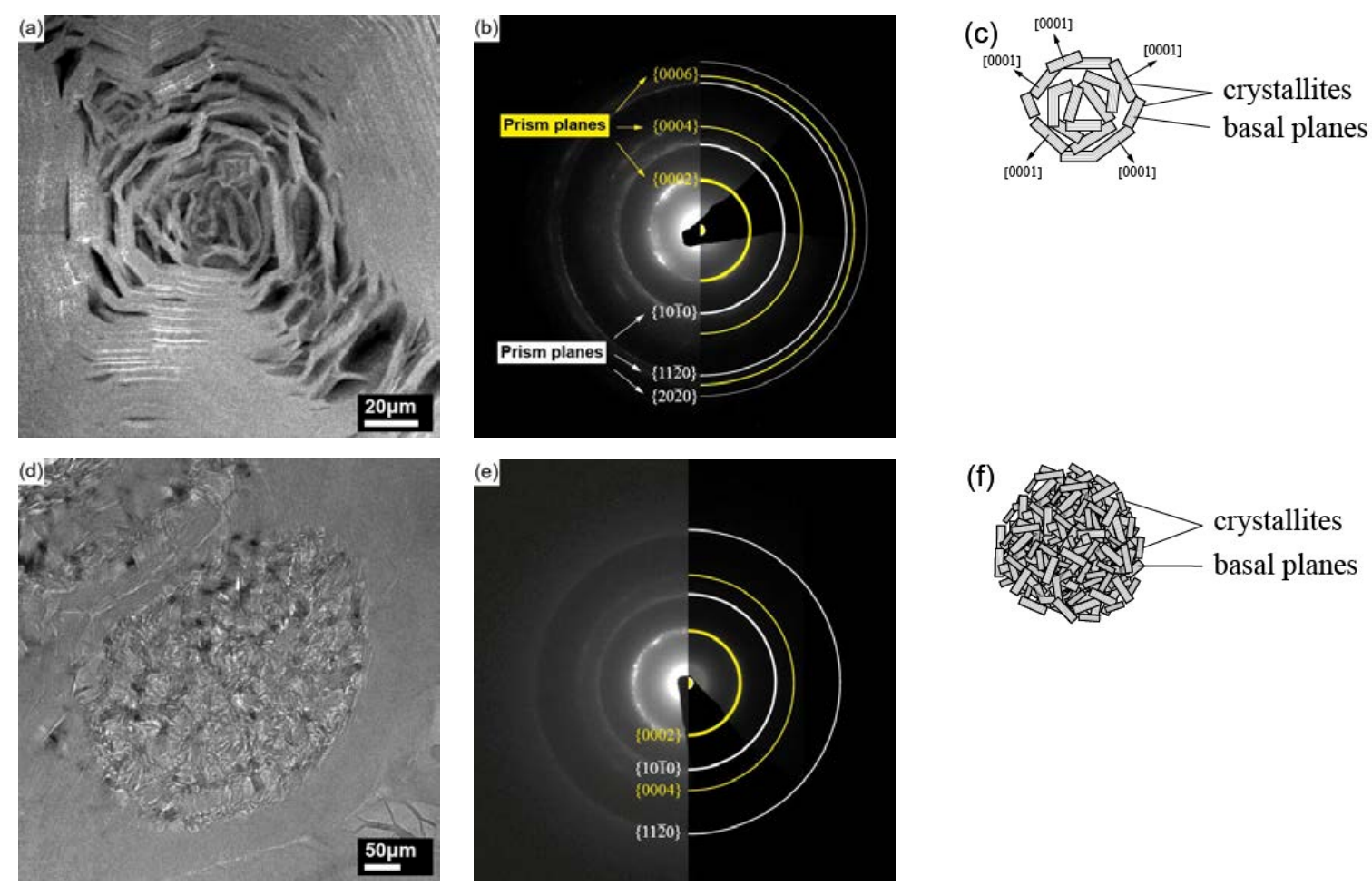

Figure 7 Structures of QI inclusions in the binding carbon region. (a) STEM ADF image of a rosette structure; (b) indexed SAED ring pattern recorded from the rosette structure in (a); (c) schematic cross-sectional view of the rosette structure of a QI inclusion; (d) TEM BF image of a chaotic structure; (e) indexed SAED ring pattern recorded from the marked region of the chaotic structure in (d); (f) schematic cross-sectional view of the chaotic structure of a QI inclusion. 
Figure 7(a) shows the detailed structure of a QI particle. The structure consists of concentrically aligned graphite nano-crystallites, and the central area itself is highly porous, as indicated by the darker contrast. The normal directions of most basal planes point to the centre of the rosette. The SAED pattern, as shown in Figure 7(b) shows diffraction rings where the $\{0002\}$ basal plane reflections contribute most of the diffraction intensity, and the higher order reflections, $\{0004\}$ and $\{0006\}$, are visible, but much weaker. It is noted that primary prismatic plane reflections are also visible with weak intensity, as indicated in Figure 7(b). We believe their presence is caused by the spherical structure of rosettes where some thin lamellae incline or are normal to the electron beam. Yamada [31] studied the rosette structure of QIs in detail, showing they are graphitised carbon blacks, which form some of the QIs found in coal-tar pitch binders [32]. The structure of the QI is schematically shown in Figure 7(c).

Apart from the rosette QI particles, often regions that are known as so-called "chaotic structures” [5], [9], [10] could be found. Unlike the rosettes, their structures do not exhibit the typical concentric, shell-like arrangement. Instead, nano-crystallites are randomly positioned and dispersed over a limited region. They are forked and can be bent in opposite directions. A schematic representation is given in Figure 7(f). Their SAED patterns are similar to those obtained from rosette structures. The number of chaotic structures in the investigated TEM specimen was determined to be approximately a tenth of that of the QI particles with rosette structure.

Inside QI particles, we have noted two types of structural features: a "sharp” bend and a "kink"-like bend. They are also seen in other regions of the graphite examined. An example of such a sharp bend region in the binding carbon is shown within the yellow square in Figure 8(a); continuously curved graphene layers are stacked to form the "sharp" bend, as shown in Figure 8(b). We examined a number of such "sharp" bends, including those in other regions of the graphite, and found that they all have the same structure consisting of continuously curved graphene layers. It is also noted that bridging ligaments exist around the tip of micro-cracks in graphite and QI particles. "Kinks” are also formed in these regions, as shown in Figure 8(c). Similar continuously bent graphene planes creating these "kinks" are shown at higher magnification in Figure 8(d). 

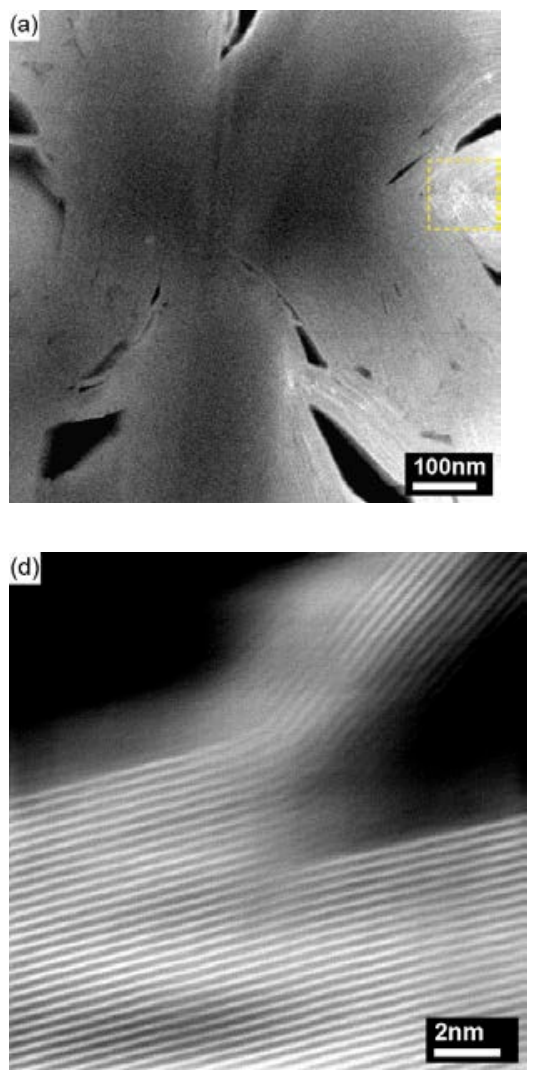
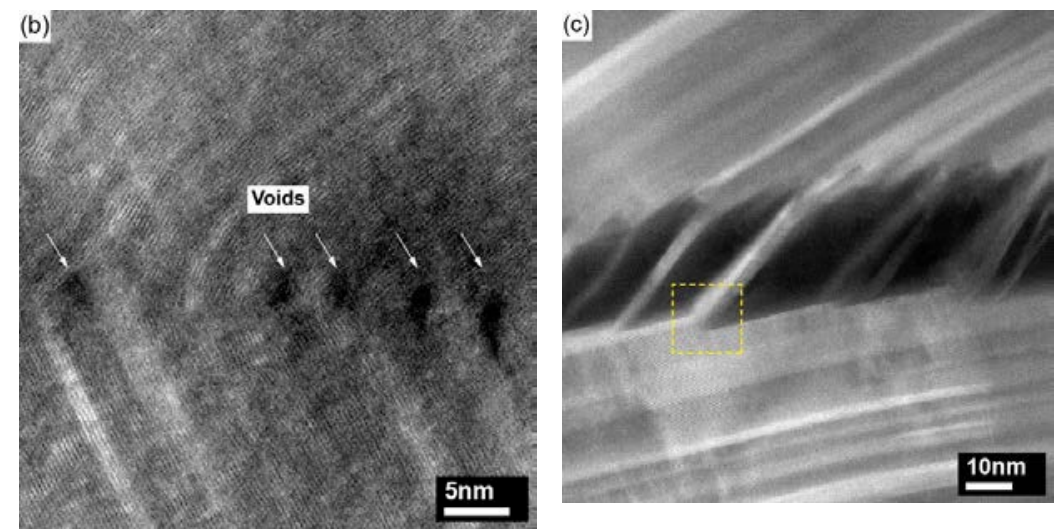

Figure 8 Detailed structure of "sharp" bent graphite and ligands linking split graphite crystals. (a) STEM ADF image showing "sharp" bent graphites in the periphery of a QI with rosette structure; (b) STEM ADF image around a "sharp" bent within the yellow square in (a); (c) STEM ADF image showing a ligand linking split graphite crystals; (d) STEM ADF image around the bend position of a ligand within the yellow square in (c).

\section{Mesoscopic structure modelling}

Our experimental observation clearly shows that the crystal structures of carbon in both coke filler and binder regions are developed into a nearly perfect graphite structure after graphitisation. However, the graphite crystallites do arrange in various formats in a 3D space after graphitisation: e.g. laminated (Figure 4), concentric (Figure 7a-c), chaotic (Figure 7d-f), spiky aligned (Figure 8a-b)) and delaminated via cracking (Figure 8c). Among these formats, three distinctive features are identified on the mesoscopic scale: in-plane boundaries between [0001] graphite crystallites with mis-aligned basal planes (Figures 4c-d); sharp bends or kinks of a graphite crystallite (Figures 8b, d) and randomly oriented boundaries between highly textured graphite domains (Figures 6e, g).

The in-plane boundaries exist in all carbon regions. Based on the TEM observation in Figure 4, we estimate that the neighbouring crystallites have an average thickness of $30 \mathrm{~nm}$ along the c-axis and a length up to $1000 \mathrm{~nm}$ in the basal plane, giving a minimum density of such boundaries around $10^{6} \mathrm{~m} / \mathrm{m}^{2}$ (the total length of boundary per unit area). If the length of the basal plane dimension $\left(\mathrm{L}_{\mathrm{a}}\right)$ is reduced to 100 or $10 \mathrm{~nm}$, the density can increase to a level of $10^{7-8} \mathrm{~m} / \mathrm{m}^{2}$. On the other hand, sharp bends or kinks are often seen in QI or when microcracks are formed along the basal planes (Figure 7 and 8). QIs constitute only about $10 \%$ of the binder and bridges are often formed near the tips of micro-cracks. Thus, the density of 
bends/kinks is significantly smaller than that of the in-plane boundaries, and this is also true of randomly oriented boundaries because they exist at an even larger scale, typically microns.

We believe these in-plane boundaries may have profound impact on the performance of graphite when subjected to neutron and ion irradiation damage. An illustration of this characteristic structure is shown in Figure 9(a), which shows a so-called "crazy paving" structure. The term 'crazy paving' is used as this describes the arrangement of slabs (typically stone) of irregular size and shape that are laid in a haphazard manner. Direct imaging of the atomic structure of the boundaries between adjacent slabs is not yet available. The main challenge is to prepare TEM samples thinner than the $\mathrm{L}_{c}$ values, $\sim 30 \mathrm{~nm}$, of the laminae, where the normal direction of the thin foil is close to the c-axis. Here, we adapt a computational method to predict and see the possible atomic structure, as described below.
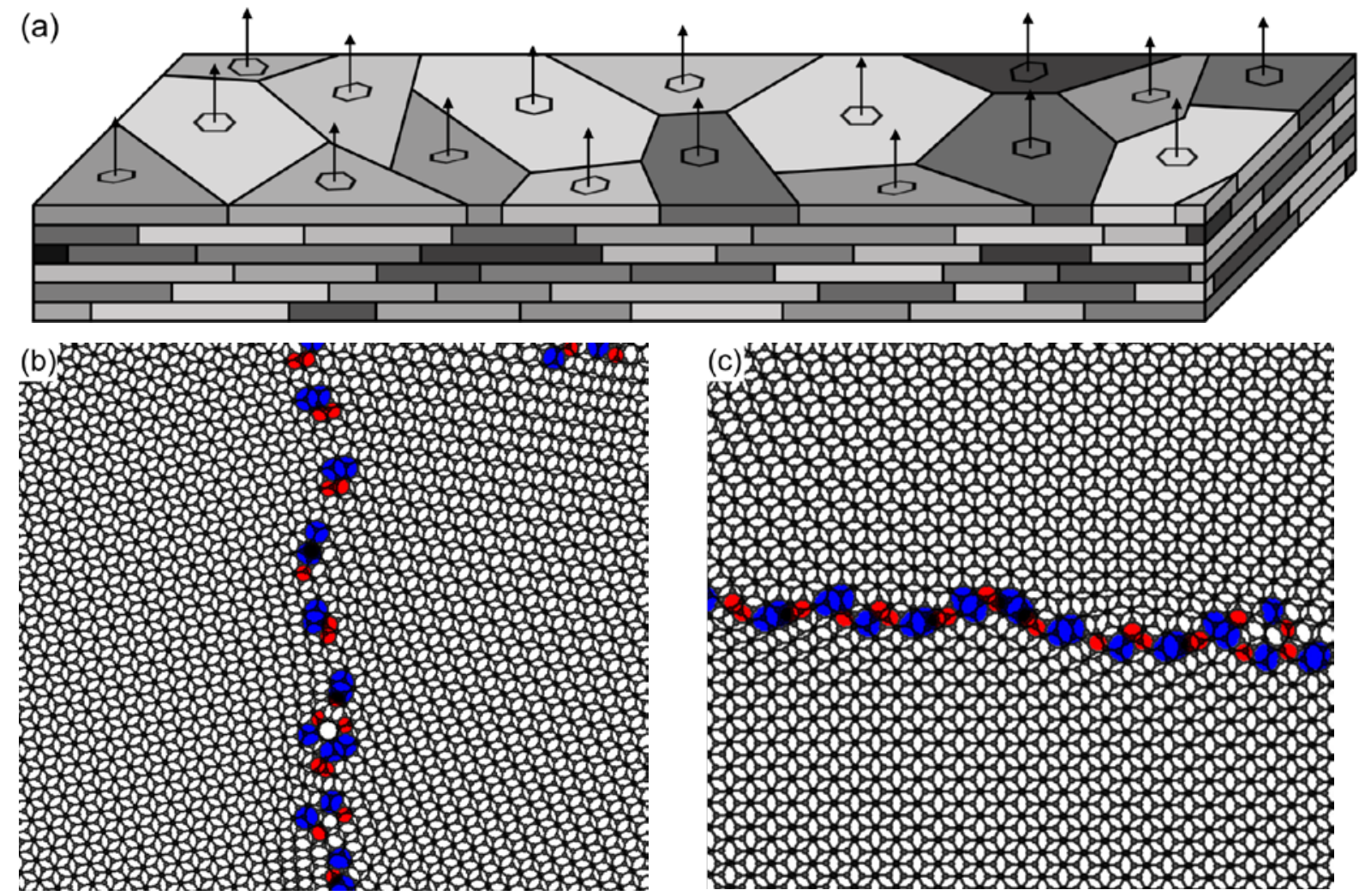

Figure 9 (a) Schematic of the laminated graphite crystallites, referred to as the "crazy paving” structure in the text. Each graphite slab has the same [0001] direction, but a random rotation is applied around this [0001] direction. Grain boundaries occur at the edges of each slab, as well as basal mis-orientation between the layers. The slab shading indicates the rotation about [0001]. (b) An example of a low angle grain boundary (6 degrees), and (c) a high angle grain boundary (35 degrees) are shown, with the 'a' and 'b' layers visible. The 7 member carbon rings are coloured blue and the 5 member carbon rings are coloured red.

Computational methods have recently been used to generate nano-crystalline graphene sheets [33]. Using similar methods, randomly orientated graphene grains were generated by uniformly distributing a set of random points in a plane. A Voronoi construction of the 2D 
plane was then generated from these points. A randomly orientated graphene grain is placed within each Voronoi cell, and the grain boundaries relaxed with the geometric centroidal Voronoi tessellation (CVT) method [34]. Further relaxation was then performed in molecular dynamics (MD) [35]. All MD simulations were performed using the Large-scale Atomic/Molecular Massively Parallel Simulator (LAMMPS).

To generate nano-crystalline graphite slabs, i.e. the crazy paving structure shown in Figure 9(a), an original computer code [36], [37] was modified to produce alternating 'ab' layers of nano-crystalline graphene to generate a nano-crystalline graphite structure [38]. This was achieved by displacing the centres of the Voronoi cells by one C-C bond length along the appropriate axis. The Voronoi construction then resulted in grain boundaries which had roughly similar locations. Simulations using the AIREBO [35] potential and a recent Reactive Force Field (ReaxFF) potential [39] were considered in this work. The ReaxFF LAMMPS simulations made use of the 'reax/c' and 'fix qeq/reax' commands [40].

Due to the hexagonal symmetry, the mis-orientation can only vary between 0 and 60 degrees. Figure 9 (b) \& (c) shows an example of a low and high angle mis-orientation respectively. The structures contain only 5, 6 and 7 member carbon rings, and all atoms have 3-fold coordination, as shown in the figures. See the original work [34] for a comprehensive analysis of the range of grain boundaries and their favourable comparison to experimental results. In the Figure 4 in the $\mathrm{DiB}$ [18], two examples of a triple junction are shown that are composed of only 5, 6 and 7 member rings.

A di-interstitial defect, which consists of a ring of 5 and 7 member carbon rings around a central 6 member carbon ring, can occur during relaxation of the grain boundaries. An example of this defect can be seen in Figure 9(b). This sub-optimal defect is a local minimum, but a lower energy global minimum can be found by removing two atoms, and relaxing the system again.

Using the AIREBO [35] potential with LAMMPS, a large slab structure $(40 \times 40 \times 3 \mathrm{~nm}$, 575527 atoms) containing 8 randomly orientated Voronoi grains, was annealed at $1000 \mathrm{~K}$ in the npt ensemble at zero pressure, as shown in Figure 10(a). During the simulation, all atoms remained in their 3-fold coordination states. However, ridges along the grain boundaries were observed. Typically, 5 member carbon rings were observed at the peak of the ridges, as shown in Figure 10(b). The pentagons are expected to cause this curvature, since they are a 
positive wedge disclination which causes this positive Gaussian curvature. This curvature is also seen in fullerenes, which contain pentagon rings. The 6 member carbon rings in the centre of the junction in Figure 10(b) are distorted from their hexagonal shape in the bulk (including strong out of plane distortions), indicating a weak point of high strain.

A recent ReaxFF potential has also been fitted to perfect and defective graphite structures [39]. After performing the initial relaxation of the geometrically determined structure and removal of atoms that were closer than a cut-off separation of $0.08 \mathrm{~nm}$, with the ReaxFF potential, similar grain structures as above were found.

(a)

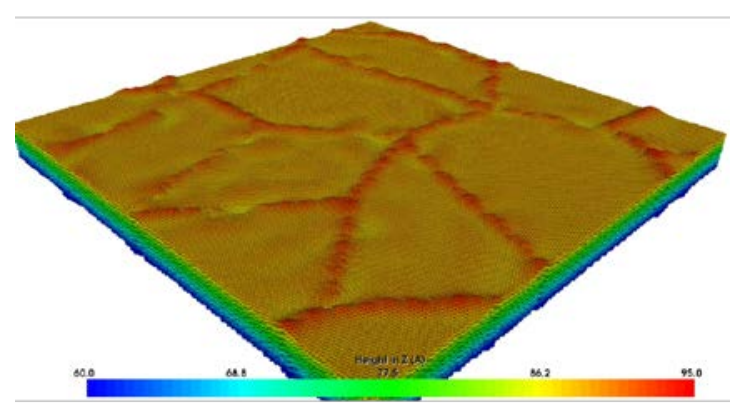

(b)

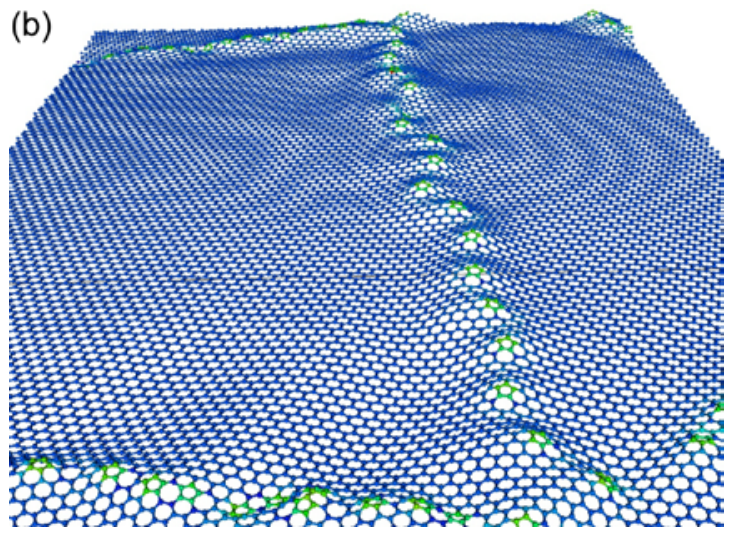

(c)

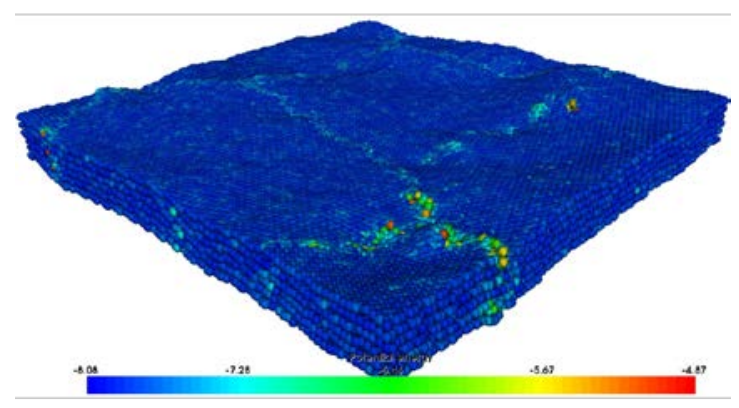

(d)

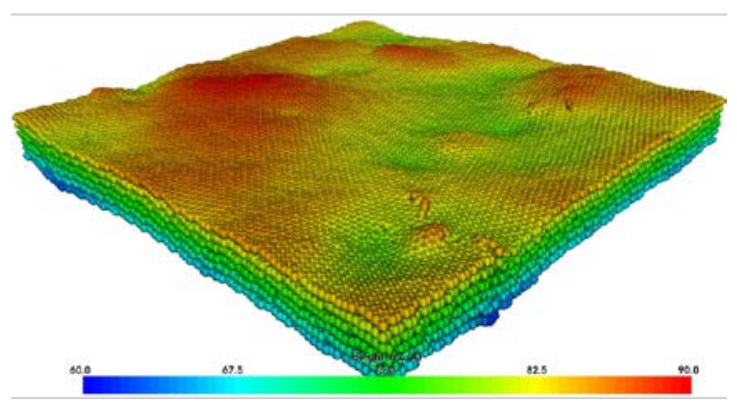

Figure $10 \quad$ (a) Graphite slabs (40x40x3 nm system with 8 randomly orientated Voronoi grains), relaxed with the AIREBO potential. (b) Only the top layer of atoms is shown, with the 5 member rings highlighted. (c-d) Graphite slabs (20x20x2 nm system with 3 randomly orientated Voronoi grains), relaxed with the ReaxFF potential. Images show a snapshot after $20 \mathrm{ps}$ of annealing at $1000 \mathrm{~K}$.

A smaller structure (20x20x2 nm, 3 Voronoi grains) was produced and annealed at $1000 \mathrm{~K}$ for 20 ps, with the Nosé-Hoover thermostat, using the ReaxFF potential. As seen in Figure 10 (c-d), the surface roughens, but there is significantly less distortion of the surface around the grain boundaries using this potential. Interestingly, several surface areas reconstructed by ejecting a small number of atoms. This indicates that the boundary may still be over dense, 
(containing too many di-interstitial defects), for this potential and a greater cut-off may be required in the geometric relaxation method that generated the grain boundary structures.

The in-plane boundary structure between graphite slabs is expected to be relaxed by 5, 6 and 7 member carbon rings. From the images shown in Figure 9-10, it is clear that the 7 member carbon rings open up the interface structure, giving arm-chair edge structure on the boundary, which is believed to represent the main contributor to the strength of the D peak in the Raman spectrum of graphite. Therefore, the estimated $\mathrm{L}_{\mathrm{a}}$ based on an $\mathrm{I}_{\mathrm{D}} / \mathrm{I}_{\mathrm{G}}$ ratio, or the measured inplane length from a TEM diffraction contrast image, should have a strong link with the existence of 7 member carbon rings.

Sharp kink boundaries were also studied, and results are shown in Figure 5 in the DiB [18]. It was found that the structure appears stable, with only minor rounding of the sharp bend. This is in good agreement with the experimental observation of these kink boundaries. This idealised structure did not contain any defects, nor did any form during MD. All atoms remained 3-fold coordinated within 6 member hexagonal rings.

\section{Discussion}

We have demonstrated that isostatically moulded graphite, SNG623, has similar structural features in coke fillers and binding carbon as those identified in previous investigations [5][9]. The present study has also shown that graphite crystal laminae are the primary constituents across all fillers and binding carbon structures. Such laminae had been previously exposed in TEM diffraction contrast images [4], [5], [7]-[9], but no further attention had yet been drawn to them. In her early study of crystallite growth in graphitising carbon based on X-ray diffraction/scattering, Franklin [41] proposed that the laminate structure of graphite is pre-defined by the graphite crystallites developed during carbonisation of hydrocarbon compounds. These crystallites retain enough mobility with weak crosslinking and tend to lie nearly parallel to one another. It is this near-parallelism of neighbouring crystallites that contribute to the crystallite growth during graphitisation through movement of whole layers. Our measured in-plane dimensions of laminae in SNG623 vary from tens to hundreds, even a thousand nanometres, with a thickness around tens of nanometres. In general, a coke filler is composed of predominantly larger laminae. In binding carbon, the dimensions of the laminae have a broad distribution, but smaller sizes are predominant. The in-plane dimensions of these laminae are directly measured from 
diffraction contrast TEM images, but can also be estimated from $\mathrm{I}_{\mathrm{D}} / \mathrm{I}_{\mathrm{G}}$ ratios of Raman spectra on a fracture surface, as fully evidenced in this study.

The ratio of D to G Raman band heights is strongly associated to the edge of graphene layers. Here we used atomistic modelling to elucidate the atomistic structure of the boundaries between aligned graphite crystallites. The boundaries are composed of 5, 6 and 7 member carbon rings. It is believed that the 7 member carbon rings form arm-chair type edges of graphene layers that contribute most to the $\mathrm{D}$ band intensity in a Raman spectrum. We believe Raman spectroscopy can be a useful tool to quantify the dimensions of laminae in graphite, in particular after irradiation when vacancies and new interstitial layers have formed.

The turbostratic structure in graphite has been widely proposed to be an important 'mesoscopic structure' in previous research. For this structure, the graphene layers may randomly translate relative to each other, and rotate about the normal of a graphene layer. However, there is no evidence from our study that turbostratic structures exist within the investigated samples, away from the grain boundaries. Nevertheless, the interface region close to the basal planes between different laminae does not necessarily follow the AB type graphite stacking. This was observed in the simulations although direct microscopic imaging of this region was not possible. In the model, the centres of the "crazy paving" structures were $\mathrm{AB}$ stacked, so this deviation from $\mathrm{AB}$ stacking could be just grain boundary effect. To clarify if a turbostratic structure exists within each lamina or not, further investigation is needed.

So far atomistic modelling has only been conducted to establish the atomistic structures of commonly observed features. Our aim is to use such modelling results to build the atomistic structure of an industrial graphite grade, for further investigations of irradiation damage.

\section{Conclusion}

Isostatically moulded graphite, SNG623, shows a structure characteristic of laminae, or “crazy paving” in both coke filler and binding carbon. In a laminate, the graphite crystallites have nearly parallel [0001] orientation but are rotated randomly. The thickness of a lamina is around $30 \mathrm{~nm}$, but the dimension in the basal plane varies from tens and hundreds to over a thousand nanometres, as estimated from the TEM diffraction contrast images. 
Raman spectroscopy results, used to determine disorder structure in graphite or graphene, can be interpreted in terms of the mesoscopic boundaries formed between graphite crystal laminae in the laminated graphite structure. The differences in the estimated $\mathrm{L}_{a}$ from $\mathrm{I}_{\mathrm{D}} / \mathrm{I}_{\mathrm{G}}$, between coke filler and binding carbon can be explained by different lamina dimensions. In the coke filler the laminae have large dimension along the basal plane direction, typically a few hundred nanometres, with a relatively narrow distribution. In the binding carbon, the QI and the polycrystalline graphite, have a broad range of lamina dimension from tens to hundreds nanometres.

Atomistic modelling shows that the prismatic boundary formed between two differently oriented graphite laminae is composed of 5, 6 and 7 member carbon rings. The 7 member rings, relaxed in an interface structure, establish a local armchair edge structure that is believed to be the disordered structure that contributes most to the intensity of the $\mathrm{D}$ band in the Raman spectrum.

\section{Acknowledgements}

We gratefully acknowledge the support of the EPSRC Advanced Materials for Nuclear Fission programme under UNIGRAF: Understanding and Improving Graphite for Nuclear Fission (grant number EP/M018822/1, EP/M018598/1, EP/M018679/1). The EPSRC grant, EP/K040375/1, for the 'South of England Analytical Electron Microscope' is also gratefully acknowledged. Special thanks are extended to Mr Dai Huang from Sinosteel Advanced Materials Co. Ltd. to grant researchers in UNIGRAF to access their experimental nuclear graphite grades and provide great support in preparation and supply of samples.

\section{References}

[1] G. Haag, D. Mindermann, G. Wilhelmi, H. Persicke, and W. Ulsamer, "Development of reactor graphite”, J. Nucl. Mater., vol. 171, no. 1, pp. 41-48, 1990.

[2] J.-P. Bonal, A. Kohyama, J. van der Laan, and L. L. Snead, "Graphite, Ceramics, and Ceramic Composites for High-Temperature Nuclear Power Systems”, MRS Bull., vol. 34, no. 1, pp. 28-34, 2009.

[3] S. Ishiyama, T. D. Burchell, J. P. Strizak, and M. Eto, "The effect of high fluence neutron irradiation on the properties of a fine-grained isotropic nuclear graphite", $J$. Nucl. Mater., vol. 230, no. 1, pp. 1-7, 1996.

[4] S. Ragan and H. Marsh, "Science and technology of graphite manufacture”, J. Mater. Sci., vol. 18, no. 11, pp. 3161-3176, 1983.

[5] K. Y. Wen, T. J. Marrow, and B. J. Marsden, "The microstructure of nuclear graphite binders”, Carbon, vol. 46, no. 1, pp. 62-71, 2008.

[6] K. Wen, J. Marrow, and B. Marsden, "Microcracks in nuclear graphite and highly oriented pyrolytic graphite (HOPG)”, J. Nucl. Mater., vol. 381, no. 1-2, pp. 199-203, 2008.

[7] A. N. Jones, G. N. Hall, M. Joyce, A. Hodgkins, K. Wen, T. J. Marrow, and B. J. Marsden, "Microstructural characterisation of nuclear grade graphite”, J. Nucl. Mater., vol. 381, no. 1-2, pp. 152-157, 2008.

[8] J. Kane, C. Karthik, D. P. Butt, W. E. Windes, and R. Ubic, "Microstructural 
characterization and pore structure analysis of nuclear graphite”, J. Nucl. Mater., vol. 415, no. 2, pp. 189-197, 2011.

[9] C. Karthik, J. Kane, D. P. Butt, W. E. Windes, and R. Ubic, "Microstructural characterization of next generation nuclear graphites”, Microsc. Microanal., vol. 18, no. 2, pp. 272-278, 2012.

[10] K. Y. Wen, J. Marrow, and B. J. Marsden, "Nanostructures of carbon in nuclear graphite”, J. Phys. Conf. Ser., vol. 126, p. 12056, 2008.

[11] K. Lafdi, S. Bonnamy, and A. Oberlin, "Tem studies of coal tars: Crude tar and its insoluble fractions”, Carbon, vol. 28, no. 1, pp. 57-63, 1990.

[12] M. S. Morgan, W. H. Schlag, and M. H. Wilt, "Surface Properties of the QuinolineInsoluble Fraction of Coal-Tar Pitch.”, J. Chem. Eng. Data, vol. 5, no. 1, pp. 81-84, 1960.

[13] C. Karthik, J. Kane, D. P. Butt, W. E. Windes, and R. Ubic, "Neutron irradiation induced microstructural changes in NBG-18 and IG-110 nuclear graphites”, Carbon, vol. 86, pp. 124-131, 2015.

[14] M. C. R. Heijna, S. de Groot, and J. A. Vreeling, "Comparison of irradiation behaviour of HTR graphite grades”, J. Nucl. Mater., vol. 492, pp. 148-156, 2017.

[15] H. J. Christie, M. Robinson, D. L. Roach, D. K. Ross, I. Suarez-Martinez, and N. A. Marks, "Simulating radiation damage cascades in graphite", Carbon, vol. 81, no. 1, pp. 105-114, 2015.

[16] M. A. Snead, A. A. Campbell, Y. Katoh, D. Huang, H. Wu, H. Yang, H. Li, T. D. Burchell, and C. Contescu, "Sinosteel AMC Graphite Irradiation Program at ORNL", in INGSM-17, Vienna, 2016.

[17] H. Yang, H. Li, L. Zhu, D. Huang, and H. Wu, "Factors Influencing Measurement Consistency of Fracture Toughness and Specimen Size Effect of Fine Grain Nuclear Grade Graphite”, in International Nuclear Graphite Specialist Meeting INGSM-17, Vienna, 2016.

[18] B. Mearz, K. Jolley, T. J. Marrow, Z. Zhou, M. Heggie, R. Smith, and H. Wu, "Mesoscopic structure of iso-graphite for nuclear applications (manuscript submitted)", Data in Brief, 2018.

[19] B. Maerz, K. Jolley, R. Smith, and H. Wu, "Subsurface damage in isostatically moulded graphite (in preparation)”, 2018.

[20] S. J. Pennycook and P. D. Nellist, Eds., Scanning Transmission Electron Microscopy. New York, NY: Springer New York, 2011.

[21] M. Lasithiotakis, B. J. Marsden, and T. James Marrow, "Annealing of ion irradiation damage in nuclear graphite”, J. Nucl. Mater., vol. 434, no. 1-3, pp. 334-346, 2013.

[22] M. A. Pimenta, G. Dresselhaus, M. S. Dresselhaus, L. G. Cançado, A. Jorio, and R. Saito, "Studying disorder in graphite-based systems by Raman spectroscopy”, Phys. Chem. Chem. Phys., vol. 9, pp. 1276-1291, 2007.

[23] L. G. Cançado, K. Takai, T. Enoki, M. Endo, Y. A. Kim, H. Mizusaki, A. Jorio, L. N. Coelho, R. Magalhães-Paniago, and M. A. Pimenta, "General equation for the determination of the crystallite size La of nanographite by Raman spectroscopy”, Appl. Phys. Lett., vol. 88, no. 16, pp. 16-19, 2006.

[24] D. S. Knight and W. B. White, "Characterization of diamond films by Raman spectroscopy”, J. Mater. Res., vol. 4, no. 2, pp. 385-393, 1989.

[25] H. M. Freeman, A. N. Jones, M. B. Ward, F. S. Hage, N. Tzelepi, Q. M. Ramasse, A. J. Scott, and R. M. D. Brydson, "On the nature of cracks and voids in nuclear graphite”, Carbon, vol. 103, pp. 45-55, 2016.

[26] G. Zheng, P. Xu, K. Sridharan, and T. Allen, "Characterization of structural defects in nuclear graphite IG-110 and NBG-18”, J. Nucl. Mater., vol. 446, no. 1-3, pp. 193-199, 2014.

[27] S. Mrozowski, "Mechanical strength, thermal expansion and structure of cokes and carbons”, Proc. 1st 2nd Conf. Carbon, pp. 31-45, 1956. 
[28] A. A. Campbell, "The Mechanism of proton irradiation-induced creep in ultra-fine grain graphite”, University of Michigan, 2014.

[29] R. Krishna, A. N. Jones, L. McDermott, and B. J. Marsden, "Neutron irradiation damage of nuclear graphite studied by high-resolution transmission electron microscopy and Raman spectroscopy”, J. Nucl. Mater., vol. 467, pp. 557-565, 2015.

[30] D. B. Williams and C. B. Carter, Transmission Electron Microscopy: A Textbook for Materials Science (Volume 1). New York: Plenum Press, 1996.

[31] K. Yamada and S. Tobisawa, "Structure and formation process of carbon blacks formed by decomposing SiC powder using a conically converging shock-wave technique”, Carbon, vol. 27, no. 6, pp. 845-852, 1989.

[32] S. S. Jones and E. M. Woodruff, "Microstructure of carbon blacks in pitch-bonded graphites and structural changes produced by gas-graphite oxidation reactions", Carbon, vol. 9, no. 3, pp. 259-264, 1971.

[33] C. Ophus, A. Shekhawat, H. Rasool, and A. Zettl, "Large-scale experimental and theoretical study of graphene grain boundary structures”, Phys. Rev. B - Condens. Matter Mater. Phys., vol. 92, no. 20, 2015.

[34] A. Shekhawat and R. O. Ritchie, "Toughness and strength of nanocrystalline graphene”, Nat. Commun., vol. 7, p. 10546, 2016.

[35] S. J. Stuart, A. B. Tutein, and J. A. Harrison, "A reactive potential for hydrocarbons with intermolecular interactions”, J. Chem. Phys., vol. 112, no. 14, pp. 6472-6486, 2000.

[36] C. Ophus and A. Shekhawat, “Atomic Structure Repository 2015.” [Online]. Available: https://sites.google.com/a/lbl.gov/atomic-structure-repository/.

[37] C. Ophus and A. Shekhawat, "Graphene.” [Online]. Available: https://github.com/ashivni/Graphene.

[38] K. Jolley, “Nanocrystalline graphite structure.” [Online]. Available: https://github.com/Kenny-Jolley/Graphene.

[39] R. Smith, K. Jolley, C. Latham, M. Heggie, A. van Duin, D. van Duin, and H. Wu, “A ReaXFF carbon potential for radiation damage studies”, Nucl. Instruments Methods Phys. Res. Sect. B Beam Interact. with Mater. Atoms, vol. 393, pp. 49-53, 2016.

[40] H. M. Aktulga, J. C. Fogarty, S. A. Pandit, and A. Y. Grama, "Parallel reactive molecular dynamics: Numerical methods and algorithmic techniques”, Parallel Comput., vol. 38, no. 4-5, pp. 245-259, 2012.

[41] R. E. Franklin, “Crystallite growth in graphitizing and non-graphitizing carbons”, Proc. R. Soc. A, vol. 209, no. 1097, pp. 196-218, 1951. 